\title{
Connection between Warburg Effect and Oncometabolites Biosynthesis with its Clinical Implications
}

\author{
Wojciech Szlasa ${ }^{1 *}$, Kamila Wala ${ }^{1}$, Aleksander Kiełbik ${ }^{1}$, Aleksandra Zalesińska ${ }^{1}$, Jolanta Saczko ${ }^{2}$ and Julita \\ Kulbacka $^{2 *}$ \\ ${ }^{1}$ Faculty of Medicine, Wroclaw Medical University, Poland
}

${ }^{2}$ Department of Molecular and Cellular Biology, Wroclaw Medical University, Poland

Submission: June 11, 2020; Published: July 13, 2020

"Corresponding author: Wojciech Szlasa and Julita Kulbacka, Faculty of Medicine and Faculty of Pharmacy, Wroclaw Medical University, Wroclaw, Poland

\begin{abstract}
Extensive biosynthesis of lactate in the glycolysis pathway, with the low efficiency of the Krebs cycle, is called the Warburg effect. The process involves the dysregulation of the glucose metabolism in the way, that it favorizes the anaerobic conditions, therefore adapting to the low oxygen saturation of the tumour. The cancer-related metabolism alternations also involve the extensive biosynthesis of oncometabolites by the cancer cells, each of which possesses various metabolic functions. For instance, the L-2- $\alpha$-hydroxyglutarate is responsible for the trapping of the Krebs cycle substrates and thus decreasing the mitochondrial glucose metabolism. That increases the effectiveness of glycolysis-the second source of the cellular ATP, thus promoting the formation of the pyruvate, that by the reduction forms the lactate. The lactate induces cellular ROS-stress by the decrease in the glutathione reductase activity.

Moreover, the cellular alternations of the glucose-related metabolism do not contribute only tumour cells, but also the cells from the cancer microenvironment. The effect includes the upregulation in lactate production and the acidification of the tumour's stroma. In this review article, the authors focused on the analysis of the relationship between cancer and glucose metabolism. The considerations include the effect of oncometabolites and Warburg effect on tumour development and progression. In the end, a short overview of the therapeutic methods for inhibition of cancer-associated glucose metabolism is described as well.
\end{abstract}

Keywords: Warburg effect; Oncometabolites; Reverse Warburg effect; Mitochondrial metabolism activity decrease

\section{Introduction}

It is widely known that glucose plays an important role in cancer growth and progression. However, the molecular bases of the process are not as clear. The combination of anaerobic metabolism with the interactions with the cancer microenvironment and overload of oncometabolites makes the process of glucose-mediated cancer genesis highly confusing [1]. Moreover, the cross-regulation of the metabolic pathways leads to the destabilization of the normal glucose equilibria by the mutation in only one enzyme. Thus, leading to the alternations in all the pathways and improper energy supply. The mutated enzymes lead to the production of oncometabolites, that alter the metabolism of the cells in various ways, for instance, the excess of the lactate decreases the redox control by glutathione reductase [2]. The changes in glucose metabolism imply the alternations in other than sugar-related metabolic pathways, like the changes in fatty acids or nucleotides synthesis [3]. In general, the altered metabolism of the cancer cell leads to the accelerated proliferation and neoplasm expansion $[4,5]$.

From the oncologic point of view, it is very important to understand the bases of the process to effectively apply the chemotherapy. Nowadays, glucose-metabolism enzymes are being used as targets for cytostatic drugs. Due to the fact, that cancer cells exhibit a higher level of glucose uptake, the drugs are more selective towards cancerous tissue without the systemic toxicity. The higher glucose accumulation in cancer cells is also used in various clinical imaging methods, like PET for instance, where the $18 \mathrm{~F}$-glucose is being administered intravenously and the drug accumulates in the tumour. By the positron annihilation reaction, 
the quant of energy could be imaged by the clinician. Therefore, understanding the relationship between cancer cells and altered glucose metabolism is crucial for effective therapy and drug design $[2,6]$.

\section{Glucose Metabolism}

Glucose metabolism is the main source of energy for the cells to function. In general, the catabolism involves the enzymes assisted decomposition of the sugars into smaller molecules, that all eventually transform into acetyl-Coenzyme A. After the glucose flows into the cell, it is being phosphorylated by hexokinase (or like in liver glucokinase) and thus becomes the metabolically active form of the sugar [7]. Then it can undergo several glucosecatabolism pathways. At first the glycolysis-the process leading to the formation of pyruvate by the reactions of isomerization, second phosphorylation in $\mathrm{C}-1$ associated - $\mathrm{OH}$ group, the and the breakage into glyceraldehyde 3-phosphate and dihydroxyacetone phosphate.

Afterwards, the glyceraldehyde 3-phosphate undergoes simultaneous reaction with a phosphate group and the formation of 1,3-bisphosphoglycerate, which decomposition leads to the formation of ATP and the formation of 3-phosphoglycerate. The process cannot be directly reversed, unlike most of the others in glycolysis. Phosphoglycerate mutase eventually catalyzes the isomerization of 3-Phosphoglycerate to 2-phosphoglycerate, which undergoes the reaction catalyzed by the enolase. The product of enolase reaction-phosphoenolpyruvate is the second source of ATP in the glycolysis, which is being formed in the reaction catalyzed by pyruvate kinase. All the process leads to the formation of pyruvate, that can undergo various metabolic pathways. The most prominent is the pyruvate decarboxylation, leading to the formation of Acetyl-CoA, NADH, $\mathrm{CO} 2$ and ATP. The others consist of the formation of alanine or the reduction into lactate $[8,9]$.

The other metabolic pathways starting with glucose-6phosphate are pentose-phosphate pathway and the isomerization to glucose-1-phosphate, that eventually leads to the accumulation of the sugar in the glycogen or the conjugation of glucose with other compounds-like membrane-associated oligosaccharides, that compose the cell's glycocalyx. The energy-related process is the pentose-phosphate pathway. The process gains energy in the NADPH nucleotides in the reactions, that uses three glucose $=-6$-phosphates for the total synthesis of NADPH, two glucose equivalents and one equivalent of glyceraldehyde 3-phosphate. It is being composed of two phases: irreversible and reversible.

The first one delivers the NADPH and leads to the decarboxylation of the hexoses to ribulose-5-phosphate, which starts the reversible phase. The second part of the pentosephosphate pathway is reversibly catalyzed by aldolase-the enzyme transferring three-carbon fragments of the phospho-sugars and ketolase-the enzyme transferring two-carbon fragments of sugars. The aim of the second phase is the reconstitution of glycolysis-associated carbohydrates-fructose-6-phosphate and glyceraldehyde-3-phosphate $[9,10]$. Curiously, the intermediateproduct of the pathway-xylulose-5-phosphate is regulating the CREBP (carbohydrate response element-binding protein)-the transactivator for the glucose-related enzymes, thus cross-linking pentose phosphate pathway with glycolysis and Krebs cycle [11].

The acetyl group-formed in the pyruvate decarboxylation reaction, is being oxidized in the mitochondria to the carbon dioxide in the process known as the Krebs cycle. The cycle involves the addition reaction of acetyl-CoA into oxalate, leading to the formation of citric acid. The acid isomerizes and forms isocitrate, that undergoes the decarboxylation to the $\alpha$-ketoglutarate with the simultaneous formation of NADH. The last compound reacts with Coenzyme A and $\mathrm{NAD}^{+}$in the reaction that leads to the decarboxylation to succinyl-CoA and NADH. Then occurs the formation of GTP and the formation of succinate. The compound is being dehydrogenized by the enzyme involving FAD for its functioning. Thus, $\mathrm{FADH}_{2}$ is being synthesized. Then the addition of water molecule occurs and malate forms. The last molecule loses the electrons and hydrogen from the carbon associated with a hydroxyl group and oxaloacetate forms. This ends the cycle and another Acetyl-CoA can undergo the Krebs cycle [12].

The energy from the cycle comes from the high-energy nucleotide molecules- $\mathrm{NADH}$ and $\mathrm{FADH}_{2}$. These are the cofactors of the enzymes associated with the electrons transport chain in the inner mitochondrial membrane [13]. The system is being composed of three complex proteins-complex I, III and IV in the membrane and one soluble-complex II. Two low mass molecules carry the electrons from one protein complex to the other. Coenzyme Q-a terpene lipophilic molecule transfers two electrons in one carrier from complex I to complex III. Cytochrome c - a low-mass protein transports one electron at once from complex III to complex IV. Complex II is the succinate dehydrogenase-the enzyme responsible for the dehydrogenation in the Krebs cycle. Each of the membrane-associated complexes has the ability of protons transfer throughout the inner mitochondrial membrane to the periplasmatic region, increasing the concentration of the hydrogen cations in the compartment.

The ions flow is an active-energy-demanding process, that uses the energy from the electrons flow in the chain. Eventually, the electrons are being transferred to the oxygen molecule and water is being formed. The energy accumulated in the potential energy across the membrane is being used to produce ATP in the process catalyzed by ATP synthase. The enzyme consists of the rotor and the transmembrane channel connected. The inflow of the $\mathrm{H}+$ ions to the inner mitochondrial compartment leads to the rotation of the rotor and simultaneous release of ATP into the periplasmatic compartment. Then ATP is being delivered to the cytoplasm by the nucleotide transporter in the outer mitochondrial membrane. The process of ATP formation with the use of the electron transfer chain is being called oxidative phosphorylation $[14,15]$. 


\section{Oncometabolites}

Oncometabolites is the term referred to the products of glucose metabolism, that has been altered by cancer. Abnormalities in metabolic processes are the result of mutations in genes encoding enzymes participating, among others, in the Krebs cycle (e.g. isocitrate dehydrogenase, succinate dehydrogenase) $[16,17]$. These compounds possess oncogenic capabilities and can accelerate tumorigenesis by the switch in cell phenotype $[18,19]$. Moreover, the cancer-related molecules can increase the rate of epithelial-mesenchymal transition (EMT) as well as contribute to the epigenetic dysregulation of the metabolism of the cells increasing the changes in cancer cells [20-22]. Importantly, EMT is a factor that increases the invasiveness of cancer cells and the possibility of infiltrating healthy tissues and promoting metastasis [22].

The pro-oncogenic functions of the molecules rise from interfering several biochemical pathways and therefore cellular processes. One of the most prominent factors, owing to the cancer transformation of the cells is the ability of the oncometabolites to decrease the cellular concentration of $\alpha$-ketoglutarate, thus interfering the $\alpha$-ketoglutarate dependent dioxygenases, like the ones engaged in proteins hydroxylation in collagen or L-carnitine biosynthesis [23-26]. Moreover, the enzymes are also responsible for epigenetic transitions, by catalyzing the methylation of DNA and histones $[16,24,25]$. In the case of this study, the most important seems to be their role as the energy sensors of the cell's metabolism. The ability leads to the further dysregulation of the cell undergoing cancer transformation.

Interestingly, the oncometabolites does not only mean the different compounds to the ones found in the normal cell, but rather the improper concentrations of the naturally occurring ones [17]. For example, L-2-hydroxyglutarate is mostly not abundant in normal cells, but fumarate and succinate are the standard components of the Krebs cycle-only in their inadequate concentration they are referred to as oncometabolites. The most abundant alternations, leading to their biosynthesis, include a mutation in genes IDH1, IDH2 (responsible for the biosynthesis of mutated isocitrate dehydrogenase) [27] or inactivating mutations in SDH and FH (encoding enzymes: succinate dehydrogenase and fumarate hydrolase) $[16,17]$.

Mechanism of oncometabolites action in the cell involves their interference into several metabolic pathways. At first, the accumulation of succinate and fumarate lowers the concentration of Krebs cycle's substrates and thus the cell remains in the state called pseudo-hypoxia [28]. The state leads to the acceleration in the glycolysis and lowering of the Krebs cycle rate [29]. The usage of the oxygen increases, leading to the insufficient oxygenation of the tissue. The hypoxia induces the mechanisms of neovascularization mediated by HIF- $\alpha$ factor-the cancer tissue increases the blood flow through the tumour and increases the chances of metastasis [30,31]. Another study showed that succinate at low concentrations (up to $200 \mu \mathrm{M}$ ) promotes the tumour angiogenesis, by increasing the amount of VEGF, in the way not associated with HIF-1 $\alpha$ [32].

The increased blood flow can also meet the generally high demands for glucose for the tumour growth. The role of oncometabolites in the regulation of tumour microenvironment also involves a decrease in T-lymphocytes responsiveness [33]. By increasing the acidic $\mathrm{pH}$, the lactate acid and adenosine intracellular concentrations and by the decrease of intracellular glucose, the lymphocytes undergo reprogramming and the attack to the cancer cells is attenuated $[34,35]$. Besides, lower $\mathrm{pH}$ leads to a decrease in cytokine production [36]. The increase in lactate concentration leads also to the differentiation of the monocytes from the blood flow into M2 pro-cancerous macrophages in the tissues. The macrophages elevate the rate of tumour development by secreting immunocompromising interleukins, like IL-10 and TGF- $\beta$, thus further decreasing the potency of the immune system towards neoplasms [37] (Figure 1).

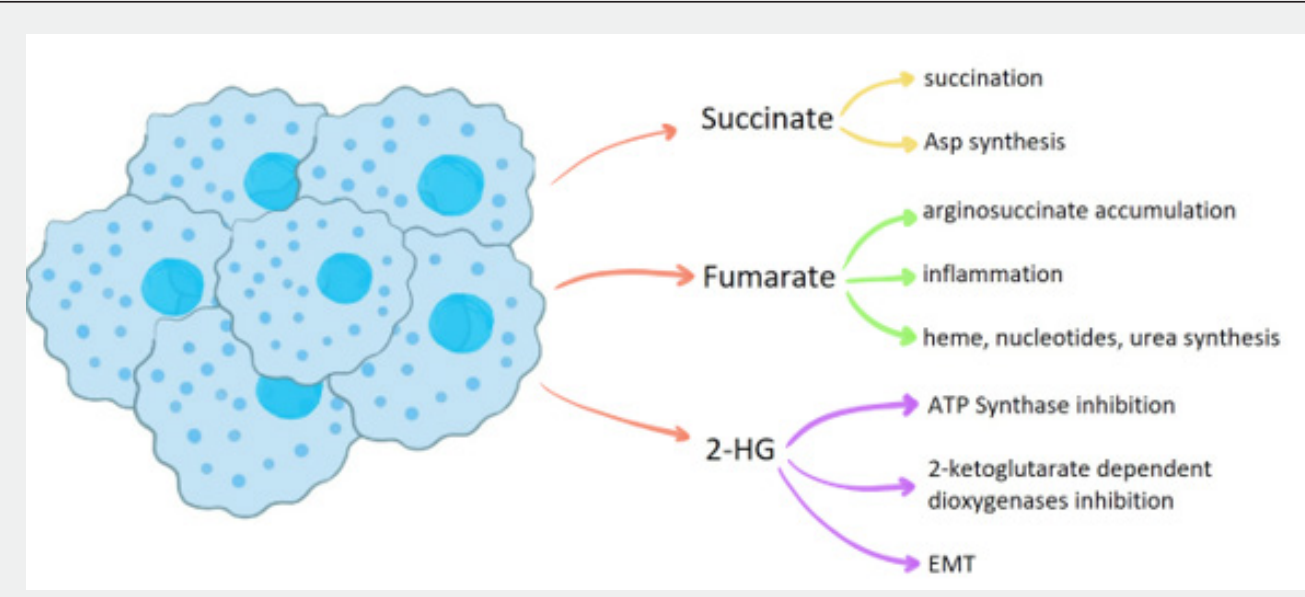

Figure 1: Oncometabolite's effects. 
Interesting seems to be the role of systemic hyperglycemia in the formation of the oncometabolites [38]. Like the cancerassociated decrease of oxaloacetate concentration for the Krebs cycle to occur, the elevated concentration of exogenous sugars also decreases the oxygen-dependent glucose catabolism by increasing the general concentration of the reduced nucleotides (NADH and
$\mathrm{FADH}_{2}$ ). This condition is caused by an increase of NADH and $\mathrm{FADH}_{2}$ production in the glycolysis pathway that occurs in a state of hyperglycemia. The accumulation of the electron's transporters leads to the lack of its free units obligatory for the Krebs cycle to work [39] (Table 1).

Table 1: Oncometabolites overview.

\begin{tabular}{|c|c|c|}
\hline Oncometabolite & Function & References \\
\hline Succinate & Increased Asp biosynthesis; succination & {$[40][18]$} \\
\hline Fumarate & $\begin{array}{c}\text { Arginosuccinate accumulation; GPx, NF-kB, NRF2 (redox signalling) and ABL-1 activation; } \\
\text { increased heme synthesis; urea cycle activity increase; nucleotides synthesis acceleration; }\end{array}$ & {$[41,42]$} \\
\hline D-2-hydroxyglutarate & $\begin{array}{c}\text { PDGFRA activation and ATP Synthase inhibition, 2-ketoglutarate dependent dioxygenases } \\
\text { inhibition - epigenetic dysregulation and pseudohypoxia response; epithelial-mesenchymal } \\
\text { transition; ferroptosis sensitization }\end{array}$ & {$[25,43,44]$} \\
\hline L-2-hydroxyglutarate & HIF-1 $\alpha$ stabilization and epigenetic changes in DNA & {$[45]$} \\
\hline
\end{tabular}

\section{Warburg Effect}

The almost total decrease of substrates flow through the Krebs cycle and the improperly elevated rate of glycolysis was called Warburg's effect-in memorial of Otto Warburg-the first researcher who described the process in detail [46]. The process generates loads of lactate while diminishing the mitochondrial activity $[46,47]$. Afterwards the biosynthesis, lactate is being transported throughout the plasma membrane and delivered to cancer's microenvironment via monocarboxylate transporters [48]. In normal cells, the pyruvate is not excreted, due to the fact, that its further metabolism continues in mitochondria. Curiously, pyruvate is an antioxidant, that helps to regulate cell's redox potential. Moreover, by the increased Krebs cycle in normal cells in comparison to the cancerous, more NADH is being formed. The electrons from the nucleotide may be transferred by transhydrogenase into NADP+, forming NADPH-a substrate for glutathione reductase, the enzyme responsible for the reduction of disulfide bonds of the cell's proteins and therefore acting against the oxidative stress [49].

Even though the cancer cells seem to be trapped in the oxidative stress damage, it only remains elevated without causing fatal damage to them. This plateau effect of highly elevated level of oxidants in the cytoplasm is caused by the hexokinase II, the cancer-related enzyme, responsible for the initiation of glucose metabolism by the phosphorylation of the sugar. Thus, Glucose6-phosphate accumulated in the cytoplasm, cannot be extensively metabolized by glycolysis but undergoes pentose phosphate shunt [50]. The pathway leads to the production of NADPH and the activity of glutathione reductase. However, the levels of NADPH in the cytoplasm remains at a lower level than compared to the normal cells, due to the high NADPH-consuming ribonucleotides reduction for the synthesis of DNA and proliferation [51].

The enzymes involved in the Warburg effect could be divided by the metabolic pathway from which they derive. From the Krebs cycle, the enzymes changed by cancer are aconitase, isocitrate dehydrogenase, succinate dehydrogenase and fumarate hydrolase [52]. The enzymes lead to the accumulation of respectively isocitrate, $\alpha$-ketoglutarate, fumarate and malate-each of which increases the pro-oncogenic process in a different way, but mostly through the decrease of free oxaloacetate molecules, inhibiting the Krebs cycle from starting [53]. In pentose phosphate shunt, only the enzymes from the irreversible phase are responsible for the increased substrates flow rate through this metabolic pathway. These include glucose-6-phosphate dehydrogenase, 6-phosphogluconate dehydrogenase, ribose-5-phosphate isomerase and ribulose-5-phosphate-3-epimerase.

This causes the up production of substrates for the reversible part of the pentose phosphate pathway, thus leading to the production of ribose for the synthesis of the nucleic acid as well as NADPH for the reduction of the ribonucleotides into deoxyribonucleotides [54]. Curiously, the metabolism of the amino acids is altered in cancer cells as well. The most important amino acid, which uptake is upregulated in various cancer cells is glutamine $[55,56]$. Being responsible for the inner mitochondrial membrane integrity, the increase in the non-essential amino acid could be considered as a biomarker of cancer cell [57]. The other amino acids, which levels are altered in cancer tissue are glycine, serine, isoleucine, leucine, and valine [58], which can be caused by the use of amino acids such as glycine and serine in gluconeogenesis [59]. It is not fully understood why some amino acids have elevated concentration in cancer tissue.

It is being hypothesized that their high content in cancer tissue is relevant to the high protein production in cancer, thus the cells must gather their high level to maintain the elevated protein's production rate. Lipid metabolism is also altered in cancer cells as well. The increased biosynthesis of the fatty acids is mainly caused by the upregulation in ATP-citrate lyase (ACL). The enzyme is responsible for linkage of Krebs cycle and fatty acids 
production by the delivery of acetyl-CoA group from citrate to the acetyl-CoA carboxylase-the rate-limiting enzyme of fatty acids synthesis $[60,61]$. Therefore, a decrease in ACL activity leads to impaired lipid synthesis, which contributes to the reduction of the risk of tumour development [62]. The elevated level of fatty acids is important in cancer cells that rely on the mitochondrial fatty acids' oxidation.

The benefits of the process over the anaerobic glycolysis are the increased ATP production and the production of NADH with $\mathrm{FADH}_{2}$ [63]. In this place could be observed the crosstalk between the cytoplasmic and mitochondrial metabolism. In some cancer cells, the fatty acid synthase is highly elevated $[64,65]$ and in the other, the fatty acid oxidation level is increased [66]. However, only the types of cancer, relying on the fatty acid's biosynthesis are considered to have the low level of mitochondrial activity $[67,68]$. At this point, there is the distinguish between the cells that use Krebs cycle to maintain high ATP levels and the cells, that rely on the glycolysis with its benefits in epigenetic transformation in cancer and high level of NADPH production, via pentose phosphate shunt, for the nucleotides production. The difference could be based on the physiological state of the cancer cell, depending if the cell needs more energy or the benefits of oncometabolites production.

The Warburg effect is the cause of many false conclusions in the therapy as well as in science. For instance, much in vitro research is based on MTT test, that directly measures the activity of mitochondrial dehydrogenases [69], not the adequate analysis of the results may potentially lead to the conclusion, that cells without the mitochondrial activity are mostly dead. However, by comparing the results from MTT to the ones obtained with flow cytometry apoptosis/necrosis studies, there could be stated that the cells are viable, but does not exhibit any mitochondrial dehydrogenases activity. The effect has been widely investigated and eventually proven to be caused by the cellular reprogramming to the hypoxic conditions of the early phases of the tumour growth. From the clinical point of view, the Warburg effect lies based on PET scan imaging. In this method, the tumour could be localized by the imaging of the radioactive conjugated glucose uptake in the body [70]. Due to the low efficiency of glycolysis in comparison to the Krebs cycle-2 ATP versus 38 ATP, the cancer cells uptake more glucose than the others [71] (Figure 2).

Figure 2: Warburg effect.

\section{Reverse warburg effect}

Reverse Warburg effect is the condition in which the fibroblasts associated with the cancer microenvironment establish the metabolic symbiosis with the cancer cells. The neoplasm releases the reactive oxygen species to the stroma of the tumour, which stimulates the glycolysis in the fibroblasts, that subsequently synthesizes the fatty acids, ketone bodies and lactate [72]. The last one is being transported through the MCT-4 transporter into the extracellular compartment [73]. Furthermore, MCT-4 expression assessment can be used to predict survival in breast cancer. The increased level of this transporter is associated with worse prognosis [74]. From there cancer cells absorb the lactate with the use of MCT-1 transporter. Further, the lactate is being used in the
NADH synthesis and after conversion to acetyl-CoA, undergoes the mitochondrial metabolism, leading to the ATP production.

The whole sequence leads to the decrease in aerobic metabolism in stromal cells and the increase of the mitochondrial metabolism in cancer [75-77]. The increase of ROS-associated stress in stromal cells also leads to the activation of the NF-kB factor, therefore promoting the inflammation. Oxidative stress leads to the upregulation of HIF- $1 \alpha$. Its elevated levels induce the nitric oxide synthase expression (NOS) and therefore promotes the vasodilation, meaning the higher blood-derived substance flow into the tumour. The factor, responsible for neovascularization, causes changes in the tumour stroma by increasing autophagy and lysosomal degradation of the cellular components. The 
degradation also relates to the loss of stromal caveolin-1, that is responsible for the suppression of tumour progression and is being the negative regulator of the Ras kinase cascade. Thus, its loss promotes tumour growth and increases the supplies of oxygen to the cancer tissue $[76,78]$ (Figure 3).

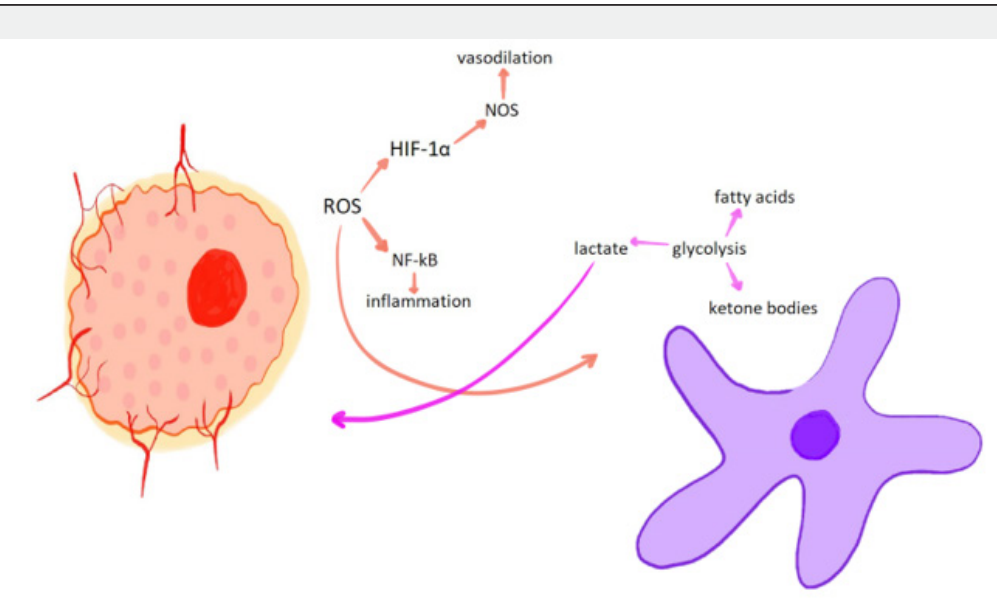

Figure 3: Reverse Warburg effect.

\section{The relationship between the Warburg effect and oncometabolites}

Even though the variety of changes among cancer cells is huge, generally all this leads to the same point. The aspects which combine all these processes is the increased lactate concentration in the cancer cells. This could be achieved in two ways, by the increased glycolysis with the reduced Krebs cycle rate or by the induction of tumour-associated fibroblasts to production and secretion of lactate (reverse Warburg effect) [79]. The further important point in the pro-oncogenic process is the synthesis of the oncometabolites, each of which has its characteristics but in general, these promote the cancer progression as well [18]. The third metabolic property of neoplasm is the accumulation or biosynthesis of the macromolecules' building blocks, like amino acids.

The process that differs the cancer cells is the rate of oxidative phosphorylation. When the excess of fatty acids is $\beta$-oxidized, the OXPHOS remains increased [80]. However, in a case when the pentose phosphate shunt rate is increased, some part of $\mathrm{NADH}$ is being transformed by transhydrogenase into $\mathrm{NAD}^{+}$ to form NADPH-used for ribonucleotides reduction and DNA's components formation [68]. Thus, the OXPHOS rate slightly decreases in comparison to the oxidation of the fatty acids [81]. Another difference between cancers is the source of the lactate in the cytoplasm. Some increase the glycolysis rate, increasing the endogenous lactate level and the others use the reverse Warburg effect, increasing the exogenous lactate levels [74].

All the metabolic changes lead to cancer progression and tumour development by changing the genetic program to the rapid proliferation and providing the molecules needed for the process [82]. The changes also involve the inhibition of specific immune response and the induction of inflammation process in the tumour environment [83].

\section{Clinical Application}

The regulation of glucose metabolism is nowadays a sufficient method of controlling cancer growth and progression. Several chemotherapeutics-analogues of glucose metabolism intermediates can effectively inhibit specific glucose metabolism pathways. The initial step of the glucose uptake by GLUT-1 can be caused by phloretin or quercetin [84]. Novel drugs like WZB117 and STF31 are nowadays tested in clinical tests [85]. The phosphorylation of the cytoplasmic glucose by hexokinase could be inhibited with 3 -bromopyruvate $[84,86]$. Further steps of glycolysis, like the reaction catalyzed by phosphofructokinase, could be deprived with the novel small-molecule drugs, such as 3PO or more potent PFK15 [87]. Treatment of cancer with combination therapy with PFK15 and metformin seems to be promising, positive results were obtained in studies on multiple myeloma cells [88].

Not only the enzymes directly involved in the glucose metabolism can be inhibited, but also the regulatory enzymes are a proper target for the cancer pharmacotherapy. The most prominent example is the inhibition of pyruvate dehydrogenase kinase with dichloroacetate $[89,90]$. There could also be found drugs that selectively inhibit the lactate biosynthesis and thus decreasing the phenotypic expression of cancer hallmarks. These include the inhibitors of lactate dehydrogenase, like oxalic acid or NHI1 [91,92]. Aside from the direct inhibition of glucose metabolism, there could be inhibited the enzymes involved in the downstream effects of glucose metabolism. For instance, HIF-1 $\alpha$ can be destabilized using digoxin or the pyrimidine biosynthesis rate could be decreased with leflunomide [93]. Attempts are also being made to modify the mutated forms of the IDH1 and IDH2 
enzymes. Several pharmacological substances such as AGI-5198 and AG-221 (Enasidenib) are under study. AGI-5198, which is an IDH1 inhibitor, reduces the level of D-2-hydroxyglutarate and inhibits the growth of glioma [94] (Figure 4).

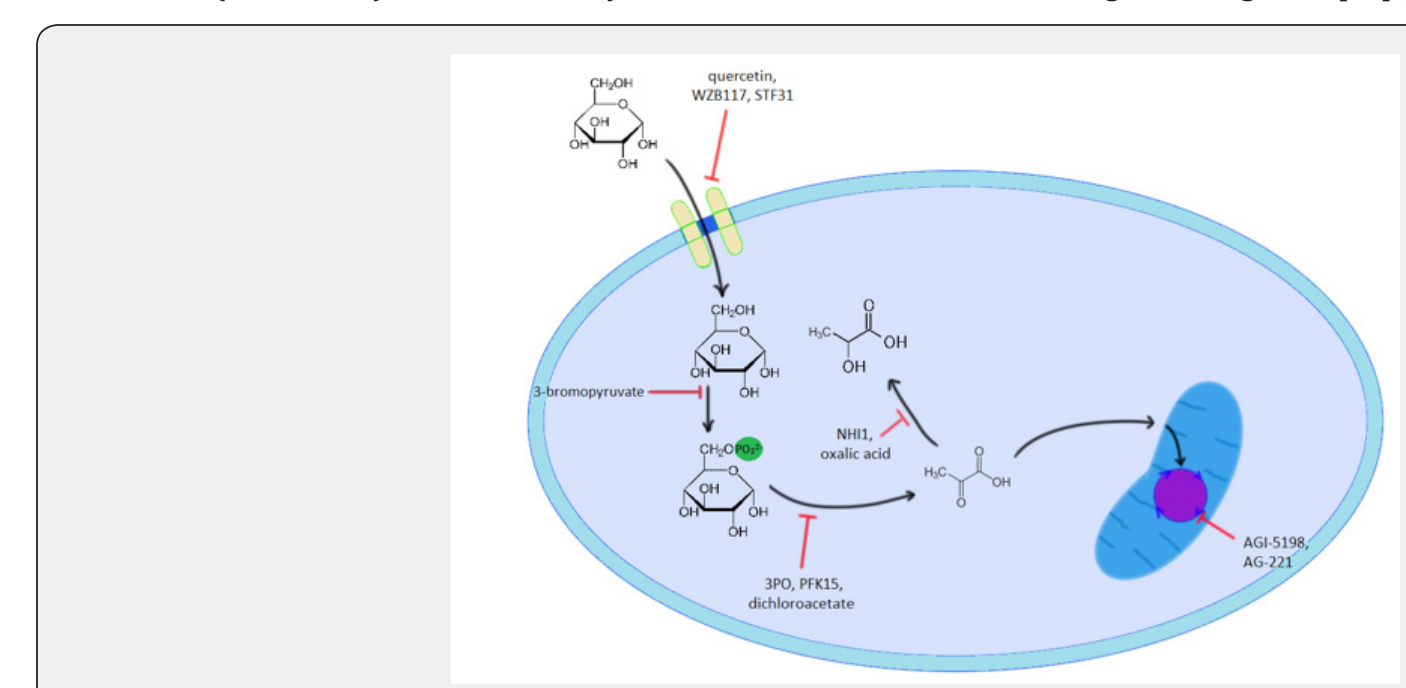

Figure 4: Clinical inhibition of Warburg effect and oncometabolites biosynthesis.

\section{Conclusion}

By the production of oncometabolites, cancer enables the tumour progression. Due to the induction of neovascularization, alternations in redox potential, cooperation with the tumourstromal cells and the accumulation of energy sources and building blocks, cancer overcomes the difficulties set by the immune system of the affected organism and the genetically programmed inhibition of mitosis and thus can develop. However, the advancement in molecular biology, drug design and clinical efforts deliver new chemotherapeutics, that could inhibit the pathological process. The only matter is to deeply understand the process one aims to target.

\section{Declaration}

Founding - This research was funded by National Science Centre (Poland) within a framework of SONATA BIS 6 No.: 2016/22/E/NZ5/00671; PI: J. Kulbacka and partially Subsidy Funds of Department of Molecular and Cellular Biology SUB. D260.20.009.

\section{Ethics Approval}

All procedures performed in studies involving human participants were in accordance with the ethical standards of the institutional and/or national research committee and with the 1964 Helsinki Declaration and its later amendments or comparable ethical standards. The study was approved by the Bioethics Committee of the Medical University of Wroclaw.

\section{Acknowledgement}

All authors contributed to the study conception and design. Material preparation, data collection and analysis were performed by Wojciech Szlasa, Kamila Wala, Aleksander Kiełbik, Aleksandra
Zalesińska, Julita Kulbacka and Jolanta Saczko. The first draft of the manuscript was written by Wojciech Szlasa and all authors commented on previous versions of the manuscript. All authors read and approved the final manuscript.

\section{References}

1. Hsu PP, Sabatini D M (2008) Cancer cell metabolism: Warburg and beyond. Cell 134: 703-707.

2. Hay N (2016) Reprogramming glucose metabolism in cancer: Can it be exploited for cancer therapy? Nat Rev Cancer 16: 635-649.

3. Weinberg F, Chandel NS (2009) Mitochondrial metabolism and cancer. Annals of the New York Academy of Sciences 1177(1): 66-73.

4. Dang CV (2012) Links between metabolism and cancer. Genes Dev 26: 877-890.

5. Annibaldi A, Widmann C (2010) Glucose metabolism in cancer cells. Curr Opin Clin Nutr Metab Care 13: 466-470.

6. Hamanaka RB, Chandel NS (2012) Targeting glucose metabolism for cancer therapy. J Exp Med 209: 211-215.

7. Jiang G, Zhang BB (2003) Glucagon and regulation of glucose metabolism. Am J Physiol-Endocrinol Metab 284(4): E671-8.

8. Turner J F, Turner DH (1980) The Regulation of Glycolysis and the Pentose Phosphate Pathway. In Metabolism and Respiration, Academic press, Elsevier, pp. 279-316.

9. Pelicano H, Martin DS, Xu RH, Huang P (2006) Glycolysis inhibition for anticancer treatment Oncogene 25: 4633-4646.

10. Patra KC, Hay N (2014) The pentose phosphate pathway and cancer. Trends Biochem Sci 39: 347-354.

11. Kabashima T, Kawaguchi T, Wadzinski BE, Uyeda K (2003) Xylulose 5-phosphate mediates glucose-induced lipogenesis by xylulose 5-phosphate-activated protein phosphatase in rat liver. Proc Natl Acad Sci U S A 100: 5107-5112.

12. Schroeder MA, Atherton HJ, Ball DR, Cole MA, Heather LC, et al. (2009) Real-time assessment of Krebs cycle metabolism using hyperpolarized 13 C magnetic resonance spectroscopy. FASEB J 23: 2529-2538. 
13. Alberts B, Johnson A, Lewis J (2002) How Cells Obtain Energy from Food - Molecular Biology of the Cell - NCBI Bookshelf In Molecular Biology of the Cell.

14. Papachristodoulou D, Snape A, Elliott W, Elliott D (2014) Biochemistry and molecular biology. ( $\left.6^{\text {th }} \mathrm{edn}\right)$, Oxford university Press, England.

15. M Saraste (1999) Oxidative Phosphorylation at the fin de siècle. Science 283(5407):1488-1493.

16. Morin A, Letouzé E, Gimenez Roqueplo AP, Favier J (2014) Oncometabolites-driven tumorigenesis: From genetics to targeted therapy. Int J Cancer 135: 2237-2248.

17. Yang M, Soga T, Pollard PJ (2013) Oncometabolites: Linking altered metabolism with cancer. J Clin Invest 123: 3652-3658.

18. Sciacovelli M, Frezza C (2016) Oncometabolites: Unconventional triggers of oncogenic signalling cascades. Free Radic Biol Med 100 175-181.

19. Nowicki S, Gottlieb E (2015) Oncometabolites: Tailoring our genes. FEBS J 282: 2796-2805

20. Colvin H, Nishida N, Konno M, Haraguchi N, Takahashi H, et al. (2016) Oncometabolite D-2-Hydroxyglurate Directly Induces EpithelialMesenchymal Transition and is Associated with Distant Metastasis in Colorectal Cancer. Sci Rep 6: 1-11.

21. Aspuria PJP, Lunt SY, Väremo L, Vergnes L, Gozo M, et al. (2014) Succinate dehydrogenase inhibition leads to epithelial-mesenchymal transition and reprogrammed carbon metabolism. Cancer Metab 2: 21.

22. Guerra F, Guaragnella N, Arbini A A, Bucci C, Giannattasio S, et al. (2017) Mitochondrial dysfunction: A novel potential driver of epithelial-tomesenchymal transition in cancer. Front Oncol 7: 295.

23. Shim EH, Livi CB, Rakheja D, Tan J, Benson D, et al. (2014) L-2 hydroxyglutarate: An epigenetic modifier and putative oncometabolite in renal cancer. Cancer Discov 4: 1290-1298.

24. Karlstaedt A, Zhang X, Vitrac H, Harmancey R, Vasquez H, et al. (2016) Oncometabolite D-2-hydroxyglutarate impairs $\alpha$-ketoglutarate dehydrogenase and contractile function in rodent heart. Proc Natl Acad Sci U S A 113: 10436-10441.

25. Xu W, Yang H, Liu Y, Yang Y, Wang P, et al. (2011) Oncometabolite 2-hydroxyglutarate is a competitive inhibitor of $\alpha$-ketoglutaratedependent dioxygenases. Cancer Cell 19: 17-30.

26. Fan J, Teng X, Liu L, Mattaini KR, Looper RE, et al. (2015) Human phosphoglycerate dehydrogenase produces the oncometabolite D-2hydroxyglutarate. ACS Chem Biol 10: 510-516.

27. Ward PS, Cross JR, Lu C, Weigert O, Abel Wahab O, et al. (2012) Identification of additional IDH mutations associated with oncometabolite R (-)-2-hydroxyglutarate production. Oncogene 31: 2491-2498.

28. Yang M, Pollard PJ (2013) Succinate: A new epigenetic hacker. Cancer Cell 23: 709-711.

29. Yang M, Soga T, Pollard PJ, Adam J (2012) The emerging role of fumarate as an oncometabolite. Front Oncol 2: 85.

30. Lu H, Forbes RA, Verma A (2002) Hypoxia-inducible factor 1 activation by aerobic glycolysis implicates the Warburg effect in carcinogenesis. J Biol Chem 277: 23111-23115.

31. Reeves KJ, Brown NJ (2015) Hypoxia and angiogenesis: From primary tumor to bone metastasis In Bone Cancer: Primary Bone Cancers and Bone Metastases: ( $\left.2^{\text {nd }} e d n\right)$, Elsevier Inc, pp. 177-189.

32. Mu X, Zhao T, Xu C, Shi W, Geng B, Shen J, et al. (2017) Oncometabolite succinate promotes angiogenesis by upregulating VEGF expression through GPR91-mediated STAT3 and ERK activation. Oncotarget 8:
13174-13185.

33. Bunse L, Pusch S, Bunse T, Sahm F, Sanghvi K, et al. (2018) Suppression of antitumor $\mathrm{T}$ cell immunity by the oncometabolite (R)-2hydroxyglutarate. Nat Med 24: 1192-1203.

34. Haas R, Smith J, Rocher Ros V, Nadkarni S, Montero Melendez T, et al. (2015) Lactate regulates metabolic and proinflammatory circuits in control of T cell migration and effector functions. PLoS Biol 13(7): e1002202.

35. Lardner A (2001) The effects of extracellular $\mathrm{pH}$ on immune function. J Leukoc Biol 69: 522-530.

36. Jiang B (2017) Aerobic glycolysis and high level of lactate in cancer metabolism and microenvironment. Genes Dis 4: 25-27.

37. Romero Garcia S, Moreno Altamirano MMB, Prado Garcia H, Sánchez García FJ (2016) Lactate contribution to the tumor microenvironment: Mechanisms effects on immune cells and therapeutic relevance. Front Immunol 7: 52 .

38. Ramteke P, Deb A, Shepal V, Bhat MK (2019) Hyperglycemia associated metabolic and molecular alterations in cancer risk progression treatment and mortality. Cancers (Basel) 11: 1402.

39. Yan LJ (2014) Pathogenesis of chronic hyperglycemia: From reductive stress to oxidative stress. J Diabetes Res 137919.

40. Eijkelenkamp K, Osinga TE, Links TP, van der Horst Schrivers ANA (2020) Clinical implications of the oncometabolite succinate in SDHxmutation carriers. Clin Genet 97: 39-53.

41. Yang M, Soga T, Pollard PJ, Adam J (2012) The emerging role of fumarate as an oncometabolite. Front Oncol 2: 85.

42. Kulkarni RA, Bak DW, Wei D, Bergholtz SE, Briney CA, et al. (2019) A chemoproteomic portrait of the oncometabolite fumarate Nat Chem Biol 15: 391-400

43. Seok J, Yoon SH, Lee SH, Jung JH, Lee YM (2019) The oncometabolite d-2-hydroxyglutarate induces angiogenic activity through the vascular endothelial growth factor receptor 2 signaling pathway. Int J Oncol 54: 753-763.

44. Wang TX, Liang JY, Zhang C, Xiong Y, Guan KL, et al. (2019) The oncometabolite 2-hydroxyglutarate produced by mutant IDH1 sensitizes cells to ferroptosis. Cell Death Dis 10: 1-12.

45. Ye D, Guan KL, Xiong Y (2006) Metabolism Activity and Targeting of Dand L-2-Hydroxyglutarates. Trends in Cancer 4: 151-165.

46. Kim JW, Dang CV (2006) Cancer's molecular sweet tooth and the warburg effect. Cancer Res 66: 8927-8930.

47. Liberti MV, Locasale JW (2015) The Warburg Effect: How Does it Benefit Cancer Cells? Trends Bio chem Sci 41: 211-218.

48. Goodwin ML, Gladden LB, Nijsten MWN, Jones KB (2015) Lactate and Cancer: Revisiting the Warburg Effect in an Era of Lactate Shuttling. Front Nutr 1-27.

49. Valko M, Rhodes CJ, Moncol J, Izakovic M, Mazur M (2006) Free radical's metals and antioxidants in oxidative stress-induced cancer. Chem Biol Interact 160: 1-40.

50. Chen Z, Zhang H, Lu W, Huang P (2009) Role of mitochondria-associated hexokinase II in cancer cell death induced by 3-bromopyruvate. Biochim Biophys Acta-Bioenerg 1787: 553-560.

51. Tedeschi PM, Markert EK, Gounder M, Lin H, Dvorzhinski D, et al. (2013) Contribution of serine folate and glycine metabolism to the ATP NADPH and purine requirements of cancer cells. Cell Death Dis 4: e877-e877.

52. Gaude E, Frezza C (2014) Defects in mitochondrial metabolism and cancer. Cancer Metab 2: 10. 
53. Mullen AR, Hu Z, Shi X, Jiang L, Boroughs LK, et al. (2014) Oxidation of alpha-ketoglutarate is required for reductive carboxylation in cancer cells with mitochondrial defects. Cell Rep 7: 1679-1690.

54. Wamelink MMC, Struys EA, Jakobs C (2008) The biochemistry metabolism and inherited defects of the pentose phosphate pathway: A review. J Inherit Metab Dis 31: 703-717.

55. Smolková K, Ježek P (2012) The role of mitochondrial NADPHdependent isocitrate dehydrogenase in cancer cells. Int J Cell Biol 12.

56. Kim MH, Kim H (2013) Oncogenes and Tumor Suppressors Regulate Glutamine Metabolism in Cancer Cells. J Cancer Prev 18: 221-226.

57. Gu Y, Chen T, Fu S, Sun X, Wang L, et al. (2015) Perioperative dynamics and significance of amino acid profiles in patients with cancer. J Transl Med 13: 35.

58. Lai HS, Lee J C, Lee PH, Wang ST, Chen WJ (2005) Plasma free amino acid profile in cancer patients. Semin Cancer Biol 15: 267-276.

59. Scioscia KA, Snyderman CH, Wagner R (1998) Altered serum amino acid profiles in head and neck cancer. Nutr Cancer 30: 144-147.

60. Currie E, Schulze A, Zechner R, Walther TC, Farese RV (2012) Cellular fatty acid metabolism and cancer. Cell Metab 18: 153-161.

61. Zhang F (2012) Dysregulated lipid metabolism in cancer. World J Biol Chem 3: 167-174.

62. Bauer DE, Hatzivassiliou G, Zhao F, Andreadis C, Thompson CB (2005) ATP citrate lyase is an important component of cell growth and transformation. Oncogene 24: 6314-6322.

63. Liu Y (2006) Fatty acid oxidation is a dominant bioenergetic pathway in prostate cancer. Prostate Cancer Prostatic Dis 9: 230-234.

64. Menendez JA, Lupu R (2017) Fatty acid synthase regulates estrogen receptor- $\alpha$ signaling in breast cancer cells. Oncogenesis 6 e299-e299.

65. Menendez JA, Lupu R (2017) Fatty acid synthase (FASN) as a therapeutic target in breast cancer. Expert Opin Ther Targets 21: 1001-1016.

66. Holla VR, Wu H, Shi Q Menter DG, DuBois RN (2011) Nuclear orphan receptor nr4a2 modulates fatty acid oxidation pathways in colorectal cancer. J Biol Chem 286: 30003-30009.

67. Samudio I, Fiegl M, Andreeff M (2009) Mitochondrial uncoupling and the warburg effect: Molecular basis for the reprogramming of cancer cell metabolism. Cancer Res 69: 2163-2166.

68. DeBerardinis RJ, Sayed N, Ditsworth D, Thompson CB (2008) Brick by brick: metabolism and tumor cell growth. Curr Opin Genet Dev 18: 54 61.

69. Supino R (1995) MTT assays. Methods Mol Biol 43: 137-149.

70. Almuhaideb Ahmad PNBJ (2011) 18F-FDG PET/CT Imaging in Oncology. Ann Saudi Med 3-13.

71. Kunkel M, Reichert TE, Benz P, Lehr HA, Jeong JH, et al. (2003) Overexpression of Glut-1 and increased glucose metabolism in tumors are associated with a poor prognosis in patients with oral squamous cell carcinoma. Cancer 97: 1015-1024.

72. Pavlides S, Whitaker Menezes D, Castello Cros R, Flomenberg N, Witkiewicz AK, et al. (2009) The reverse Warburg effect: Aerobic glycolysis in cancer associated fibroblasts and the tumor stroma. Cell Cycle 8: 3984-4001.

73. Gerlinger M, Santos CR, Spencer Dene B, Martinez P, Endesfelder D, et al. (2012) Genome-wide RNA interference analysis of renal carcinoma survival regulators identifies MCT4 as a Warburg effect metabolic target. J Pathol 227: 146-156.

74. Witkiewicz AK, Whitaker Menezes D, Dasgupta A, Philp NJ, Lin Z, et al. (2012) Using the "reverse Warburg effect" to identify high-risk breast cancer patients: Stromal MCT4 Predicts Poor Clinical Outcome in Triple-Negative Breast Cancers. Cell Cycle 11: 1108-1117.

75. Sotgia F, Martinez Outschoorn UE, Lisanti MP (2014) The reverse warburg effect in osteosarcoma. Oncotarget 5: 7982-7983.

76. Wilde L, Roche M, Domingo Vidal M, Tanson K, Philp N, et al. (2017) Metabolic coupling and the Reverse Warburg Effect in cancer: Implications for novel biomarker and anticancer agent development. Semin Oncol 44: 198-203.

77. Lee M (2015) Metabolic interplay between glycolysis and mitochondrial oxidation: The reverse Warburg effect and its therapeutic implication. World J Biol Chem 6: 148.

78. Fu Y, Liu S, Yin S, Niu W, Xiong W, et al. (2017) The reverse Warburg effect is likely to be an Achilles' heel of cancer that can be exploited for cancer therapy. Oncotarget 8(34): 57813-57825.

79. Strickaert A, Corbet C, Spinette SA, Craciun L, Dom G, et al. (2019) Reprogramming of Energy Metabolism: Increased Expression and Roles of Pyruvate Carboxylase in Papillary Thyroid Cancer. Thyroid 29(6): 845-857.

80. Jiang P, Du W, Wu M (2014) Regulation of the pentose phosphate pathway in cancer. Protein Cell 5: 1-11.

81. Baquer NZ, Hothersall JS, McLean P (1988) Function and Regulation of the Pentose Phosphate Pathway in Brain. Current Topics in Cellular Regulation. Academic Press 29: 265-289.

82. Ferreira LMR (2010) Cancer metabolism: The Warburg effect today. Exp Mol Pathol 89: 372-380.

83. Allavena P, Mantovani A (2012) Immunology in the clinic review series; focus on cancer: Tumour-associated macrophages: Undisputed stars of the inflammatory tumour microenvironment. Clin Exp Immunol 167: 195-205.

84. Chen Z, Lu W, Garcia Prieto C, Huang P (2007) The Warburg effect and its cancer therapeutic implications. J Bioenerg Biomembr 39: 267-274.

85. Kraus D, Reckenbeil J, Veit N, Kuerpig S, Meisenheimer M, et al. (2018) Targeting glucose transport and the NAD pathway in tumor cells with STF-31: a re-evaluation. Cell Oncol 41: 485-494.

86. Lis P, Dylag M, Niedźwiecka K, Ko YH, Pedersen PL, et al. (2016) The HK2 dependent "Warburg effect" and mitochondrial oxidative phosphorylation in cancer: Targets for effective therapy with 3-bromopyruvate Molecules 21: 1730.

87. Zhu W, Ye L, Zhang J, Yu P, Wang H, et al. (2016) PFK15 a small molecule inhibitor of PFKFB3 induces cell cycle arrest apoptosis and inhibits invasion in gastric cancer. PLoS One 11(9): e016376.

88. Liu X, Zhao Y, Zhang E, Yan H, Lv N, et al. (2019) The synergistic effect of PFK15 with metformin exerts anti-myeloma activity via PFKFB3. Biochem Biophys Res Commun 515: 332-338.

89. Strum SB, Adalsteinsson Ö, Black RR, Segal D, Peress NL, et al. (2013) Case report: Sodium dichloroacetate (DCA) inhibition of the "warburg Effect" in a human cancer patient: Complete response in non-Hodgkin's lymphoma after disease progression with rituximab-CHOP. J Bioenerg Biomembr 45: 307-315.

90. Papandreou I, Goliasova T, Denko NC (2011) Anticancer drugs that target metabolism: Is dichloroacetate the new paradigm? Int J Cancer 128: 1001-1008.

91. Le A, Cooper CR, Gouw AM, Dinavahi R, Maitra A, et al. (2010) Inhibition of lactate dehydrogenase A induces oxidative stress and inhibits tumor progression. Proc Natl Acad Sci U S A 107: 2037-2042.

92. Farah IO, Lewis VL, Ayensu WK, Cameron JA (2013) Assessing the survival of MRC-5 and A549 cell lines upon exposure to honey and D-glucose. Biomed Sci Instrum 49: 101-108. 
93. Shukla SK, Purohit V, Mehla K, Gunda V, Chaika NV, et al. (2017) MUC1 and HIF-1alpha Signaling Crosstalk Induces Anabolic Glucose Metabolism to Impart Gemcitabine Resistance to Pancreatic Cancer. Cancer Cell 32: 71-87.
94. Luengo A, Gui DY, Vander Heiden MG (2017) Targeting Metabolism for Cancer Therapy. Cell Chem Biol 24: 1161-1180.

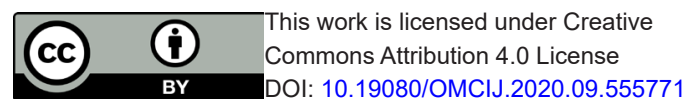

Your next submission with Juniper Publishers will reach you the below assets

- Quality Editorial service

- Swift Peer Review

- Reprints availability

- E-prints Service

- Manuscript Podcast for convenient understanding

- Global attai nment for your research

- Manuscript accessibility in different formats

( Pdf, E-pub, Full Text, Audio)

- Unceasing customer service

Track the below URL for one-step submission https://juniperpublishers.com/online-submission.php 